\title{
An improved ASM matching method
}

\author{
Cai-Xian Liao \\ School of IOT Engineering, Jiangnan University \\ Jiangnan University \\ Wuxi city, Jiangsu province, China \\ liaocaixian0601@126.com
}

\begin{abstract}
The traditional active shape model (ASM), according to the principal component analysis, gets main components for the shape, and then the average shape model is obtained to approximately express target object model. However, when the difference between the feature shape model of target and the average shape model is large, it is difficult to match. Therefore, this paper analyses the shortage of the traditional matching algorithm, and proposes an improved algorithm. This algorithm searches the most similar image to the target object from the training sample set, and uses the shape model of the most similar image instead of the average shape model to approximately express target object model, so it can avoid the failure caused by the large difference between the feature shape model of the target and the average shape model.
\end{abstract}

Keywords-ASM; average shape; matching algorithm; similar image

\section{INTRODUCTION}

With the rapid development of the artificial intelligence and the pattern recognition, the technology related to the face is being used widely, especially the face recognition technology. As a field of study with wide applications, the face recognition technology attracts a large number of the domestic and foreign researchers to participate in. The ideal face recognition technology is very mature, but a lot of problems also need to be solved to develop truly robust, large-scale, and fast face recognition system [1].

The active shape model (ASM) is a model for the face recognition which is used widely, is proposed by T.F.Cootes in 1995 [2]. In ASM, face shapes are modeled by point distributions models (PDMs). The points of a PDM are landmark points, and the location of these landmark points on face images can have location (or matching) errors. An ASM for faces is trained based on a face image training set, and then the aligned points are used to represent the shape of each face. Each PDM landmark point represents a relevant facial feature location (e.g., eye centers, face boundaries, etc.), and the visual aspect (appearance) of that landmark point in the face image is described by a feature set (e.g., chrominance, texture, etc.)[5].

Because the traditional ASM model uses the average shape model to approximately express the shape of target, and then matches the target object by limiting the variable shape parameters. However, when the difference between the feature shape model of target and the average shape model is large, the effect of the matching algorithm is not ideal.

\author{
Xiao-Jun $\mathrm{Wu}$ \\ School of IOT Engineering, Jiangnan University \\ Jiangnan University \\ Wuxi city, Jiangsu province, China \\ xiaojun_wu_jnu@163.com
}

In order to solve this problem, this paper proposes a solution: we search the most similar image to the target object from the training sample set, and use the shape model of the most similar image instead of the average shape model to approximately express target object model, and then match the target object by limiting the variable shape parameters. So it can avoid the failure caused by the large difference.

This paper is arranged as following: the second section introduces the improved ASM matching algorithm; The third section analyzes and compares the experimental results; Finally conclusions are drawn in section four..

\section{AN IMPROVED ASM MATCHING ALGORITHM}

This section proposes an improved ASM matching algorithm based on the traditional ASM algorithm.

\section{A. The traditional ASM algorithm}

The main idea of the traditional ASM algorithm is shown in Fig.1:

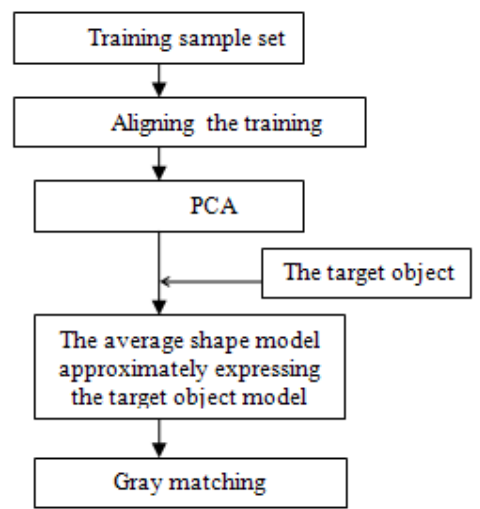

Figure 1. The main idea of the traditional ASM algorithm

Assuming that the sample $\Omega$ has $N$ training image samples, marking $m$ feature points manually, the training sample set and the face image can be expressed by a shape vector [2] :

$$
\begin{aligned}
& \Omega=\left\{s_{1}, \cdots, s_{i}, \cdots, s_{N}\right\} \\
& s_{i}=\left(x_{i 1}, y_{i 1}, \cdots, x_{i j}, y_{i j}, \cdots x_{i m}, y_{i m}\right)^{T}
\end{aligned}
$$

where $s_{i}$ means the $i$-th training sample, $\left(x_{i j}, y_{i j}\right)$ is the coordinate of the $j$-th feature point of the $i$-th training sample. 
1) Aligning the training set: After obtaining the training sample set $\Omega$, it is found that the distribution of the feature points is very confused, and it absolutely has no face shape, but the fuzzy face outline can be seen. Therefore the training sets need to be aligned. This article uses the Procrustes algorithm [3], the basic idea is to minimize the sum of the distances from the all shapes to the average shape. Assume that $M(S c, \theta)[s]$ is a transformation to $S$ with the rotation angle $\theta$ for the rotational change and the $S c$ for the scale. Therefore:

$$
M(S c, \theta)\left[\begin{array}{l}
x_{i j} \\
y_{i j}
\end{array}\right]=\left(\begin{array}{l}
S c^{*} \cos \theta * x_{i j}-S c * \sin \theta^{*} y_{i j} \\
S c * \sin \theta * x_{i j}+S c^{*} \cos \theta^{*} y_{i j}
\end{array}\right)
$$

The aligning process from $S_{2}$ to $S_{1}$ is actually a process to solve the rotation angle $\theta$, scale $S c$ and the displacement $\left(t_{x}, t_{y}\right)$.The solution to these parameters is given as the following equations. Details are given in the reference [3].

$$
\left[\begin{array}{cccc}
X_{2} & -Y_{2} & W & 0 \\
Y_{2} & X_{2} & W & 0 \\
Z & 0 & X_{2} & Y_{2} \\
0 & Z & -Y_{2} & X_{2}
\end{array}\right]\left(\begin{array}{c}
S c * \cos \theta \\
S c * \sin \theta \\
t_{x} \\
t_{y}
\end{array}\right)=\left(\begin{array}{c}
X_{1} \\
Y_{1} \\
C_{1} \\
C_{2}
\end{array}\right)
$$

where:

$$
\begin{aligned}
X_{i} & =\sum_{k=1}^{n} w_{k} x_{i k} \\
Y_{i} & =\sum_{k=1}^{n} w_{k} y_{i k} \\
Z & =\sum_{k=1}^{n} w_{k}\left(x_{2 k}^{2}+y_{2 k}^{2}\right) \\
W & =\sum_{k=1}^{n} w_{k} \\
C_{1} & =\sum_{k=1}^{n} w_{k}\left(x_{1 k} x_{2 k}+y_{1 k} y_{2 k}\right) \\
C_{2} & =\sum_{k=1}^{n} w_{k}\left(y_{1 k} x_{2 k}-x_{1 k} y_{2 k}\right)
\end{aligned}
$$

After getting the rotation angle $\theta$, scale $S c$ and the displacement $\left(t_{x}, t_{y}\right)$, then the training sets can be updated by

$$
s_{i}=M(S c, \theta)\left[s_{i}\right]+\left(t_{x}, t_{y}, t_{x}, t_{y}, \cdots, t_{x}, t_{y}\right)
$$

where $i=1,2, \ldots N$.

2) The principal component analysis: After alignment to the face images in the training set, the statistical rule for the shape change can be found by using the PCA method. Details are given in the reference [4].

a) Calculate the average shape:

$$
\bar{S}=\frac{1}{N} \sum_{i=1}^{N} \tilde{S}_{i}
$$

b) Calculate the covariance matrix:

$$
\operatorname{Cov}=\frac{1}{N-1} \sum_{i=1}^{N}\left(\tilde{s_{i}}-\bar{s}\right)\left(\tilde{s_{i}}-\bar{s}\right)^{T}
$$

c) Find out the eigenvalue $\lambda$ and the eigenvector $p$ of the $\operatorname{Cov}$, choose the largest $r$ eigenvalues $\left[\lambda_{1}, \lambda_{2}, \cdots, \lambda_{r}\right]$ such that.

$$
\frac{\sum_{i=1}^{r} \lambda_{i}}{\sum \lambda_{i}} \geq \eta
$$

d) Choose the eigenvectors $P=\left[p_{1}, p_{2}, \cdots, p_{r}\right]$ corresponding to the largest $r$ eigenvalues, the statistical model of the training set can be obtained by:

$$
s=\bar{s}+P b
$$

3) Gray matching: The average shape model approximately expresses the target object, the optimal matching points are obtained by calculating Markovian, and then the various parameters can be updated by

$$
d b=P^{T} d x
$$

where $d x$ is the displacement, $d b$ is the change of shape parameter.

\section{B. The improved ASM matching algorithm}

The main idea of the improved ASM algorithm is shown in Fig.2:

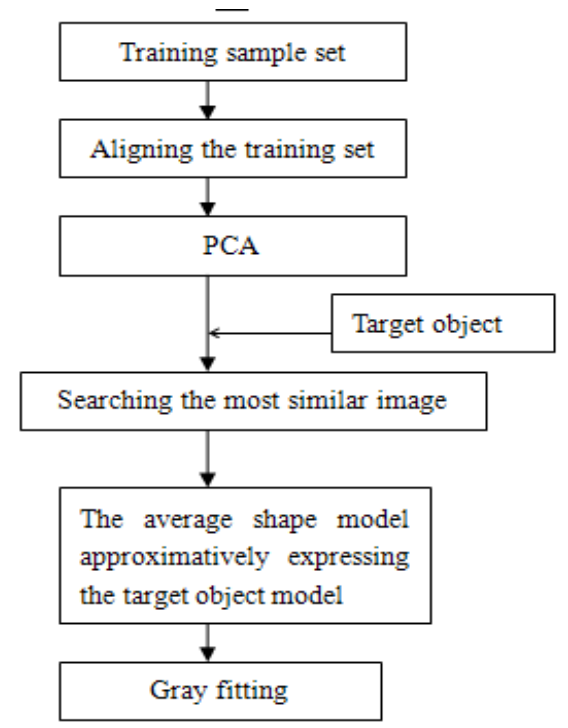

Figure 2. The main idea of the improved ASM algorithm 
Compared with the traditional ASM model algorithm, the improved algorithm adds a process of searching the most similar image to the target object from the training sample set, and uses the shape model of the similar image instead of the average shape model to approximately express target object model. Now, searching the most similar image process will be introduced in detail.

1) Search the former $z$ points whose gray value is the most similar to the i-th feature point of the j-th image in the area with this feature point as the center and the distance being $\mathrm{d}$.Take $\mathrm{d}=2, \mathrm{z}=9$ for example.

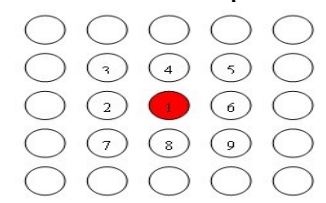

Figure 3. Similar points calculation

where the red point is the $i$-th feature point of the $j$-th image. The positions of the nine points marked above are just assumed points.

2) Calculate the mean gray value of these $z$ points, marked as $d g_{i j}$. We know there are $m$ feature points in each image, the gray information set $d g_{j}$ of the $\mathrm{j}$-th image could be regained.

$$
d g_{j}=\left[d g_{1 j}, \cdots d g_{i j}, \cdots d g_{m j}\right]^{T}
$$

where $j \in\{1,2, \cdots, N\}, N$ is the number of the training images.

3) Get the gray value for the feature points of each image projecting in the target image, so the gray matrix could be gained.

$$
G=\left[g_{1}, \cdots, g_{j}, \cdots g_{N}\right]
$$

where $g_{j}$ is the gray vector of the characteristic points of the $j$-th training image projecting in the target image.

4) If the target image shape model is very closed to some image shape model in the training set, the Euclidean distance will be small. In order to find the most similar image to the target image, we only need to solve the following problem.

$$
i_{0}=\underset{j}{\arg \min }\left(\left(d g_{j}-g_{j}\right)^{T}\left(d g_{j}-g_{j}\right)\right)
$$

The $i_{0}$-th image gotten in the formula (19) is the most similar image. So, we take the $i_{0}$-th image as the approximate expression to the target image.

\section{THE EXPERIMENTAL RESULTS AND ANALYSIS}

In this section, we used 36 target images to make the experiment, and all the lateral face images using the improved algorithm have nice matching results. And then we choose the following two target images in the Weizmann face database as the representative of the comparative experiments.

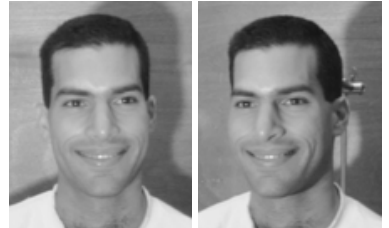

(a)

(b)

Figure 4. The target image

A. The experimental results and analysis to the target image (a)

Now we have a test to the target image (a) using the traditional ASM model algorithm.

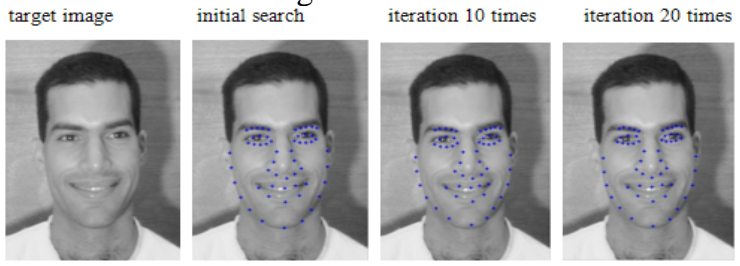

Figure 5. The result of the test to the target image (a) using the traditional ASM

It could be seen from Fig. 5 that the average shape model can express the target object model very well, that is to say, the difference between the target's feature shape model and the average shape model is small. The matching effect could be improved after 10 times of iteration but not very obvious.

Next we use the improved algorithm to do a test also to the target image (a).

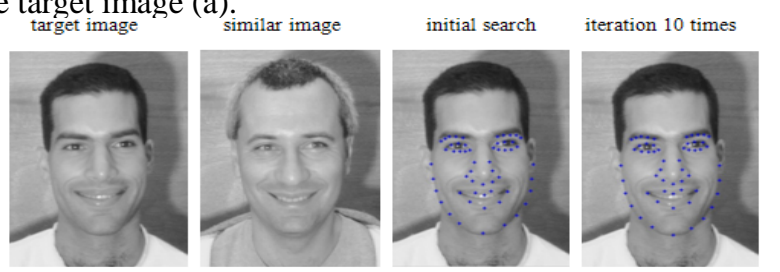

Figure 6. The result of the test to the target image (a) using the improved ASM

The improved ASM attempt to search the most similar image to the target image from the training sample set, and use the shape model of the similar image instead of the average shape model to approximately express target object model, as shown in the Fig.6.

Then we use the Euclidean distance to show the errors between the similar positions and the true positions with using the traditional method and the improved method. As shown in Table I.

TABLE I. THE ERRORS WITH THE TWO METHODS TO THE FIRST TARGET

\begin{tabular}{|c|c|c|}
\hline method & Error & Per point's error \\
\hline Traditional & 469.9708 & 8.3923 \\
\hline Improved & 441.4502 & 7.88 \\
\hline
\end{tabular}

From the Table II, it is easy to know that the errors between the similar positions and the true positions with 
using the traditional method and the improved method are similar, that is to say the improved algorithm does not get significant effect.

\section{B. The experimental results and analysis to the target image (b)}

Now we do a test to the target image (b) using the traditional ASM model algorithm.

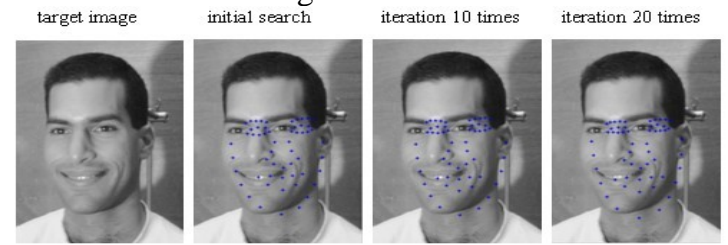

Figure 7. the result of the test to the target (b) image using the traditional ASM

The image (b) is a lateral face, so its shape model is significantly different to the average shape model. As shown in Fig.7, the average shape model seriously deviates from the target image in the initial search, and can not approximately express target image model. After 10 times of iteration, it still can not match successfully.

And now we use the improved algorithm to do the test also to the target image (b).

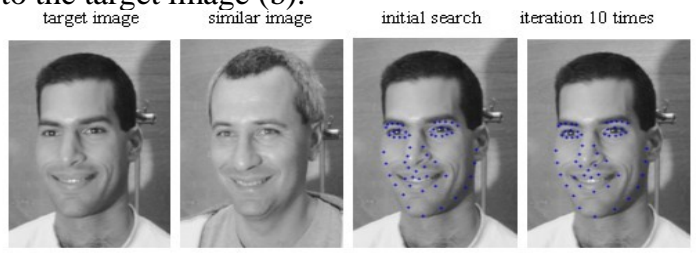

Figure 8. he result of the test to the target image (b) using the improved ASM

According to the experimental results given in the Fig.8, it can be seen that the improved algorithm could search the most similar lateral face to the target image even though the target image is a lateral face. And then use the most similar shape model to approximately express target image model, the effect is already very well after searching the most similar image.

Then the errors between the similar positions and the true positions with using the traditional method and the improved method are shown in Table II.

TABLE II. THE ERRORS WITH THE TWO METHODS TO THE SECOND TARGET

\begin{tabular}{|c|c|c|}
\hline method & Error & Per point's error \\
\hline Traditional & 2166.8 & 38.6933 \\
\hline Improved & 510.2188 & 9.1111 \\
\hline
\end{tabular}

From the Table II, the errors between the similar positions and the true positions with using the traditional method are apparently larger than the errors of improved method.

Comparison of the test results in the Fig.7 and the Fig.8, indicates that the improved algorithm gets significant improvement.

\section{The test results and analysis}

Through the above two experiments, two conclusions can be gained as follows.

- When the difference between the feature shape model of target object and the average shape model is small, the traditional matching algorithm can match very well, and the improved algorithm does not have significant advantage.

- When the difference between the feature shape model of target object and the average shape model is large, the effect of the traditional matching algorithm is not ideal. But the improved algorithm can overcome this problem, can still match very well.

\section{CONCLUSION}

This paper based on the traditional ASM algorithm, adds a process of searching the most similar image to the target object, and then uses the shape model of the most similar image instead of the average shape model to approximately express the target image model. So this algorithm avoids the failure caused by the large difference between the feature shape model of target object and the average shape model. The improved algorithm has no significant advantage when the difference between the feature shape model of target object and the average shape model is small, but when this difference is large, the improved algorithm can still match well but the traditional algorithm can not .

In view of the situation that searching the most similar image proposed in this paper only used the Euclidean distance to find the most similar image, the robustness is not very well. How to improve the robustness still needs to be studied in future.

\section{ACKNOWLEDGMENT}

This work was supported in part by the following projects: 111 Project of Chinese Ministry of Education (Grant No. B12018), Key Grant Project of Chinese Ministry of Education (Grant No.: 311024), National Natural Science Foundation of P. R. China (Grant No.: 60973094, 61103128), Fundamental Research Funds for the Central Universities (Grant No. JUSRP31103).

\section{REFERENCES}

[1] Zhen-Hua Feng. Face recognition technology-based on the feature extraction of ASM and AAM [D] : [master's degree thesis]. Wuxi: jiangnan university, 2009

[2] T.F.Cootes,C.J.Taylar,D.H.Cooper,J.Graham. Active shape models:their training and application [J].Computer Vision and Image Understanding,1995,61(1):38-59

[3] JollielT. Principle component analysis[M].New York:SpringerVerlag, 1986

[4] carlos A.R. Behaine and Jacob Scharcanski. Enhancing the performance of Active Shape Model in Face Recognition Applications. Transations on Instrumentation and Measurem, 2012

[5] Zhou Tao. The research of Feature Extraction Methods and Their Application [D]: [master's degree thesis]. Wuxi: jiangnan university, 2012 